for surveillance. These centres did not report any nosocomial infections during the survey timeframe.

Conclusions Response to the survey and adherence to BTS (2013) and HTM 01-06 (2016) guidelines were lower than expected. Nosocomial infection was rare but associated with significant morbidity, and occurred only where water channel sampling was not practiced. It is recommended that steps are taken to improve adherence to decontamination guidelines and include suction channel sampling for surveillance.

\section{G454(P) FLAGELLATE ERYTHEMA ASSOCIATED WITH JUVENILE DERMATOMYOSITIS}

${ }^{1} \mathrm{CH}$ Hew, ${ }^{2} \mathrm{LS}$ Solman, ${ }^{3} \mathrm{GT}$ Taylor, ${ }^{1} \mathrm{SC}$ Clark, ${ }^{3} \mathrm{TA}$ Amin, ${ }^{3} \mathrm{MW}$ Wood. ${ }^{1}$ Dermatology, Leeds Teaching Hospital NHS Trust, Leeds, UK; ${ }^{2}$ Paediatric Dermatology, Great Ormond Street Hospital, London, UK; ${ }^{3}$ Paediatric Rheumatology, Leeds Teaching Hospital NHS Trust, Leeds, UK

\subsection{6/archdischild-2018-rcpch.443}

Aim Flagellate erythema was first reported in association with bleomycin treatment and since then has been linked to chemotherapeutic agents, dermatomyositis, adult-onset Still disease and Shiitake mushroom dermatitis. To date, only one patient with juvenile dermatomyositis and flagellate erythema has been reported in the literature. Hence, we would like to present this case and highlight importance of this uncommon dermatological condition in children.

Method A 9-year-old girl presented in 2016, with a 2 month history of new onset proximal muscle weakness, heliotrope rash, Gottron's papules, leg erythema and soft tissue restriction at elbows, hips and knees. Muscle biopsy and MRI confirmed myositis and fasciitis. Juvenile dermatomyositis was diagnosed.

She commenced steroid therapy, initially intermittent monthly pulsed intravenous methylprednisolone, with oral prednisolone, in conjunction with subcutaneous methotrexate and oral hydroxychloroquine. Two months later, due to lack of significant improvement and deterioration in muscle function, intermittent pulsed intravenous cyclophosphamide was substituted for methotrexate, with improvement in muscle weakness.

Three months into cyclophosphamide therapy, she developed a florid, widespread eruption, with violaceous, urticated erythema of the face and upper trunk with areas of sparing. There were also erythematous, linear flagellate areas on her proximal limbs and back. Dermatographism was absent. There was no associated deterioration in muscle strength or elevation of inflammatory markers or muscle enzymes at that time. No relation to medication or potential allergic exposures was noted.

Intravenous immunoglobulin therapy was commenced but unfortunately caused an anaphylactic reaction. Methotrexate was restarted and she has been maintained since on a combination of this with hydroxychloroquine, sun protection, topical steroids and calcineurin inhibitors with improvement in her skin and muscle strength and oedema on MRI.

Result Skin biopsy showed a marked vacuolar interface reaction with colloid bodies and epidermal atrophy associated with a mild perivascular lymphocytic infiltrate in the superficial dermis with absence of eosinophils and increased dermal oedema and mucin. The features were in keeping with a flagellate erythema secondary to dermatomyositis.

Conclusion The pathogenesis is not completely clear, although physical injury, sun exposure or minor trauma could be incriminated. The significance of this rash in relation to the prognosis or underlying neoplasm in adults remains unclear.

\section{G455(P) LOWER RESPIRATORY TRACT INFECTION IN PATIENTS WITH NEUROLOGICAL IMPAIRMENT: A NARRATIVE REVIEW ON MICROBIOTA AND ANTIMICROBIAL INTERVENTIONS}

1J Burgess-Shannon, ${ }^{2} \mathrm{~L}$ Thomson. 'West of Scotland Paediatric Trainee, NHS, Glasgow, UK;

${ }^{2}$ Paediatric Respiratory Medicine, NHS Greater Glasgow and Clyde, Glasgow, UK

\subsection{6/archdischild-2018-rcpch.444}

Background Lower respiratory tract infections (LRTI) are a major cause of morbidity and mortality in patients with neurological impairment. Prophylactic antibiotics and eradication of colonisers are approaches employed in the prevention of recurrent LRTI.

Aims To determine the evidence base for this practice in children with neurological impairment.

Methods An electronic database search identified studies reporting on outcomes of efficacy for antimicrobial interventions and microbial findings relevant to LRTI in our population of interest. Results were synthesised into a narrative review.

Results Our search revealed a small case-series suggesting nebulized tobramycin may be effective in reducing the frequency of pneumonias and associated hospitalisations. We identified 5 papers focusing on microbial findings - there was significant variability in the frequency of bacterial isolation, and in the species identified. These studies were heterogeneous and subject to individual biases which limits the wider applicability of results.

Conclusions The direct evidence for antimicrobial interventions as a prophylactic strategy against LRTI in patients with neurological impairment is lacking and warrants further research. In addition, we still lack a clear understanding of the microbiota contributing to respiratory pathology, which could inform therapeutic targets.

\section{G456(P) WAVING THE RED FLAG: CHARACTERISTICS OF CHILDREN WHO REQUIRE CRITICAL CARE FOR LIFE- THREATENING ASTHMA AND WHEEZE}

${ }^{1} \mathrm{SY}$ Chau, ${ }^{1} \mathrm{C}$ Brown, ${ }^{2,3} \mathrm{MN}$ Hurley. ${ }^{1}$ Paediatrics, Nottingham University Hospitals, Nottingham, UK; ${ }^{2}$ Paediatric Respiratory Medicine, Nottingham University Hospitals, Nottingham, UK; ${ }^{3}$ Division of Child Health, University of Nottingham, Nottingham, UK

\subsection{6/archdischild-2018-rcpch.445}

Aim The RCPCH report 'State of Child Health' (2017) reported the prevalence of deaths due to asthma in the UK as among the highest in Europe. Local differences in these indices suggest varying standards of care may be an underlying factor.

As part of an evaluation of the hospital-wide asthma service, we aimed to identify characteristics of children who required critical care in order to determine if the needs of this highrisk group are being met.

Method The PICU database was interrogated to identify children who had required critical care for wheeze or asthma. Characteristics of the critical care stay were retrieved from the clinical record to identify characteristics locally of children 